\title{
Razones y consideraciones para la implementación del aprendizaje móvil en la UNED: realidades y desafíos
}

Olga Ligia Brenes Matarrita'

Ileana Salas Campos ${ }^{2}$

Cinthya Valerio Álvarez ${ }^{3}$

\begin{abstract}
1. Programa de Informática Educativa. Universidad Estatal a Distancia, Costa Rica; obrenes@uned.ac.cr
2. Programa de Producción Electrónica Multimedial. Universidad Estatal a Distancia, Costa Rica; isalas@uned.ac.cr

3. Programa de Aprendizaje en Línea. Universidad Estatal a Distancia, Costa Rica; cvalerio@uned.ac.cr
\end{abstract}

\section{Resumen}

El propósito de esta investigación consistió en conocer el estado del arte sobre el aprendizaje móvil, así como determinar los aspectos técnicos y pedagógicos implicados en el proyecto de investigación titulado "Propuesta para la experimentación, diseño, desarrollo e implementación del aprendizaje móvil (mobile learning) UNED Costa Rica”. En esta investigación participan representantes del Programa de Informática Educativa, del Programa de Aprendizaje en Línea y Programa de Producción Electrónica Multimedial de la Universidad Estatal a Distancia.

La metodología utilizada se basa en los lineamientos de Galvan (2006) para las revisiones de literatura. Una primera etapa exploratoria permitió revisar, no solo los autores fundamentales para dar sustento a las ideas planteadas, sino también las instituciones que tenían experiencias e información que podía ser útil. Una segunda etapa de la investigación coadyuvó a la delimitación de contenidos y de referencias, para la construcción del documento principal de la investigación.

Los principales resultados son contundentes, en cuanto a la urgente necesidad, que tienen las instituciones de educación superior, de implementación del aprendizaje a través de dispositivos móviles, para responder a las necesidades de la presencia de situaciones académicas en cualquier momento y en cualquier lugar.

\section{Abstract \\ Reasons and considerations for the implementation of mobile learning at uned: realities and challenges}

The purpose of this research was to know the theoretical framework about mobile learning, and to determine the technical and pedagogical aspects involved in the research project entitled "Proposal for experimentation, design, development and implementation of mobile learning (aprendizaje móvil) at UNED Costa Rica". The researches belong to the Computer Education Program, Learning Program Online Electronics and Multimedia Production Program State University Distance.

The methodology is based on the guidelines of Galvan (2006) for literature reviews. A first exploratory stage allowed to review not only the fundamental authors to give support to the ideas, but also the institutions that had experience and information that could be useful. A second stage of the research contributed to the definition of content and references in order to build the main document of the research.

The main results are conclusive as to the urgent need for higher education institutions in the implementation of learning through mobile devices to meet the needs of the present academic situations anytime, anywhere.

We suggest that future researchers test the experiences resulting from the implementation of educational 
Se sugiere que futuras investigaciones partan de la evaluación de las experiencias derivadas de la implementación de recursos educativos y de gestión administrativa, accesibles desde dispositivos móviles.

Palabras clave: Aprendizaje móvil, teléfono móvil, método de aprendizaje, educación a distancia.

\section{INTRODUCCIÓN}

Desde sus inicios, y debido a su naturaleza, la Universidad Estatal a Distancia (UNED) ha requerido del uso de los medios de comunicación para llevar a cabo los procesos de enseñanza y aprendizaje. Si bien las primeras experiencias fueron incipientes, para finales del siglo XX, la UNED inició el pilotaje de herramientas para cursos en línea, producción de multimedios y videoconferencias.

A pesar de que los contextos institucionales no siempre se ubican de manera paralela al desarrollo y demandas de la docencia, las tendencias y las nuevas generaciones de estudiantes requieren incursionar en el campo de las tecnologías móviles. Actualmente, la población estudiantil y docente, cuenta con una diversidad de medios de comunicación que les permite la conexión a Internet de forma permanente y desde cualquier lugar.

Dentro de esta realidad, el Modelo Pedagógico de la UNED, exige la necesidad de que la propuesta educativa incorpore el uso de una amplia gama de medios y apoyos didácticos, considerando nuevas alternativas de comunicación cuando sea pertinente (UNED, 2004). Este documento señala que, los medios de comunicación que se establezcan, deberán garantizar "una interacción permanente, sincrónica o asincrónicamente, entre el alumno, los especialistas y los materiales didácticos, y facilitar la retroalimentación..." (UNED, 2004, p. 12). Lo anterior incita procesos más autónomos para el desarrollo del aprendizaje, por parte de los estudiantes, y ofrece, a los docentes, opciones para implementar resources and administrative management accessible from mobile devices.

Key words: Mobile learning, mobile phones, learning methods, distance education.

innovaciones pedagógicas que respondan a la diversidad de los contextos educativos en el sistema de educación a distancia.

Dadas, esta realidad y la importancia de que la universidad cuente con una propuesta para la incorporación de dispositivos móviles, la cual atienda las demandas de un modelo de quinta generación de educación a distancia, se propone el desarrollo de una investigación que lleve al diseño de un plan para la integración pedagógica de las tecnologías móviles en la UNED. Este documento es producto de la primera etapa de investigación: conocer el marco conceptual del aprendizaje móvil, así como los aspectos técnicos y pedagógicos implicados

Para abordar este tema, se dará inicio haciendo referencia a la relación que existe entre el aprendizaje ubicuo y el aprendizaje móvil, posteriormente, se abordarán las razones que justifican el uso de tecnologías móviles y los modelos de uso en contextos educativos. Además, se mencionarán algunas consideraciones para la producción de materiales para tecnologías móviles y, finalmente, se hará referencia a las consideraciones políticas y a algunos ejemplos de implementación del aprendizaje móvil.

\section{- Aprendizaje móvil, aprendizaje ubicuo}

Antes de iniciar con el desarrollo de este tema, es necesaria la revisión de algunas definiciones del concepto "dispositivos móviles". Por ejemplo, 
Smaldino, Lowther y Russel (2008) definen las tecnologías móviles como "cualquier tipo de dispositivo de computación móvil” (p. 340). En otras palabras, son todos aquellos aparatos electrónicos que cuentan con características especiales que permiten que el usuario se traslade de un lugar a otro, sin ninguna complicación. Cabe mencionar que, en ocasiones, las personas consideran solo al teléfono celular como tecnología móvil, y no se percatan de que existen otros dispositivos que trabajan bajo el concepto de movilidad; entre los más comunes están: los lapiceros digitales, las computadoras personales, las minicomputadoras, las tabletas digitales, los reproductores de audio y vídeo, las agendas electrónicas, entre otros.

Por su parte, Roquet (2008) señala que el cómputo móvil se refiere a los "equipos de información y comunicación que no obligan a los usuarios a estar conectados mediante cables a una infraestructura de red y/o a la energía eléctrica" (p. 34), agregando, a la lista de Smaldino y otros (2008), los sistemas de posición geo-referenciada.

Dada la evolución tecnológica, lo que hoy se conoce como dispositivos móviles puede variar en el futuro, por esta razón, la UNESCO (2013) ha decidido que acogerá una amplia conceptualización, en la cual se considera como tales aquellos que

son digitales, portátiles, controlados por lo general por una persona (y no por una institución), que es además su dueña, tienen acceso a Internet y capacidad multimedia, y pueden facilitar un gran número de tareas, especialmente las relacionadas con la comunicación (p. 6).

Como puede observarse en las definiciones anteriores, las características de las tecnologías móviles permiten planear y estructurar nuevas oportunidades para aprender en la vida diaria, ya sea en el hogar, el trabajo o en el ámbito social. Es, precisamente, a través del desarrollo de estas tecnologías que se generan cambios sustanciales en los métodos de comunicación, transferencia y almacenamiento de datos. Lo anterior conlleva a la creación de nuevas propuestas de aprendizaje, tales como: e-learning (aprendizaje electrónico), m-learning (aprendizaje móvil) y, más recientemente, el u-learning (aprendizaje ubicuo)
(Burbules, 2010; Jones y Jo, 2004). Si bien algunos autores establecen una diferencia entre cada uno de estos constructos, otros los integran, tal es el caso de García, Ruiz y Domínguez (2007). Para ellos, el aprendizaje móvil, o m-learning, es el resultado de la integración del e-learning con los dispositivos móviles de comunicación, lo que eventualmente favorece el aprendizaje ubicuo. Es "la posibilidad de aprender a través de Internet, pero con máxima portabilidad, interactividad y conectividad" (p. 82).

Por otro lado, Pegrum (2013) señala que el término "aprendizaje ubicuo" se ha usado de manera alternativa al m-learning $\mathrm{y}$, si bien los dos términos pueden tener puntos en común, puede decirse que el aprendizaje ubicuo enfatiza más en el contexto, en lo situado, mientras que el m-learning enfatiza en la movilidad e independencia contextual.

Partiendo de la definición de García y otros (2007) y de Pegrum (2013), el aprendizaje móvil tiene tres características:

1. Portabilidad: es la cualidad de los dispositivos que se pueden portar y operar en la mano, también llamados computadoras de mano o handheld.

2. Interactividad: es el proceso mediante el cual los estudiantes actúan con los contenidos, a través de los dispositivos.

3. Conectividad: es la posibilidad de acceso a Internet donde quiera que se esté, lo que facilita la consulta de contenidos académicos.

Esto es posible gracias a las herramientas de la Web 2.0, integradas en los dispositivos móviles que aprovechan el potencial de las redes sociales, del envío de correos electrónicos, acceso a la Red, grabación de audio y vídeo, entre otros. Por esto es que la computación móvil se convierte en un aspecto de gran apoyo, ya que provee agilidad y optimización en los procesos que se realizan.

La convergencia de tecnologías en los dispositivos móviles hace que exista una evidente tendencia a la popularización de estos aparatos, que son, a la vez, computadoras de mano, teléfonos móviles y cámaras digitales; los cuales permiten ejecutar diferentes acciones en un solo dispositivo (Enríquez 
y Chaos, 2006). Esta convergencia está asociada al exponencial crecimiento de desarrollo de aplicaciones para móviles, que, según Koekkoek (2012), se contabilizan por miles, lo cual le permite al usuario tener acceso a diversidad de posibilidades con solo un aparato.

Por otra parte, las estadísticas muestran un crecimiento en el uso de dispositivos móviles para acceder a Internet. En el caso de Costa Rica, la encuesta realizada por Unimer en el 2012, para el periódico La Nación, indica que cada día son más los usuarios que acceden a Internet desde sus celulares. El 43,9\% de la población, en Costa Rica, tiene acceso a Internet desde su dispositivo móvil, el doble de usuarios del año anterior; si bien es evidente que aún existe una brecha entre las zonas urbanas y rurales, los datos muestran una creciente cobertura (Agüero, 2012).

Como consecuencia de este crecimiento en el uso de Internet, la comunicación se ha vuelto más versátil gracias a los servicios de mensajería instantánea, lo cual favorece la inmediatez de los procesos comunicativos, permitiendo, no solo la transmisión textual, sino también el uso de imágenes y vídeos (Enríquez y Chaos, 2006). Actualmente, la comunicación utiliza diferentes formas de transmisión como Internet, línea telefónica, satelital, entre otros. Desde el punto de vista educativo, los estudiantes tendrían la posibilidad de generar o acceder a videoconferencias, clases virtuales y "capturar, procesar, almacenar y recuperar datos e incluso, enviar información a un servidor, vía Internet" (Enríquez y Chaos, 2006, párr. 7).

Todo lo anterior lleva a afirmar que los dispositivos móviles proveen muchas posibilidades tecnológicas que hacen de ellos, recursos útiles en el campo educativo. Sin embargo, Marés (2012) atribuye otros beneficios, tales como: la alfabetización, el desarrollo de destrezas cognitivas y colaborativas y, por supuesto, el impacto sobre la motivación de los estudiantes.

\section{- Modelos de uso de los dispositivos móviles en contextos educativos}

Debido al uso masivo de teléfonos inteligentes y la creciente adquisición de tabletas, Looi, Seow, Zhang, So, Chen, y Wong (citados por Pregum, 2013) proponen y defienden el uso de dispositivos móviles en la educación, basándose en el modelo uno a uno (1:1), esto es, cada estudiante usa al menos un dispositivo móvil y este, a su vez, le pertenece. La adopción de este modelo fortalecería la propuesta Bring Your Own Device (BYOD, en español: traiga su propio dispositivo) o Bring Your Own Technology (BYOT, en español: traiga su propia tecnología), esto porque sería relativamente fácil pensar que todos los estudiantes lleven a su clase, o utilicen para sus procesos de aprendizaje, al menos un teléfono inteligente que puede ser usado como herramienta, no solo en el contexto formal, sino también en el informal. En otras palabras, el contexto educativo debe sacar provecho de la tecnología con la que ya cuenta el estudiante.

Cabe resaltar que Johnson, Adams y Cummins (2012) y Marés (2012) argumentan que, es necesario que las instituciones educativas aprovechen las competencias que poseen los estudiantes; como la navegación en la Red durante espacios prolongados de tiempo y la interacción en diferentes redes sociales, para evaluar sus estrategias y optar por modelos de enseñanza alternativos, que combinen con los modelos presenciales y a distancia. El reto está en pasar a métodos de aprendizaje más activos, con el fin de aprovechar el gran potencial que tienen las tecnologías. Bajo este mismo enfoque, Pegrum (2013) insiste en la necesidad de plantear estrategias de enseñanza que aseguren que los estudiantes sean capaces de investigar, crear, comunicar, manejar y operar las tecnologías, partiendo de un uso social y ético aceptable.

Otro aspecto, que está íntimamente relacionado con las propuestas anteriores, es la necesidad de que la didáctica apoyada en dispositivos móviles se fundamente en la realidad del estudiante. El 
hecho de que la movilidad rompa los conceptos de espacio y tiempo demanda comprender cómo puede convertirse la vida cotidiana en "espacios para el aprendizaje", esto implica expandir la visión de lo que entendemos como clase y currículo. Una visión centrada en el contexto particular de cada estudiante tiene como consecuencia un aprendizaje más personalizado, sustentado, aún más, en las tendencias de uso de entornos personales para el aprendizaje (PLEs, Personal Learning Environments), de redes personales para el aprendizaje (PLNs, Personal Learning Networks) y del uso de e-portafolios (Pegrum, 2013).

Pegrum (2014) indica que partiendo de tres elementos fundamentales: dispositivos, educandos y experiencias de aprendizaje, se pueden distinguir tres tipos de agendas o modelos para el uso de dispositivos móviles en contextos educativos, a saber:

- Tipo 1. Dispositivos en clase, pero experiencias y estudiantes no son móviles. Su agenda es la transformación de la propuesta pedagógica. Se trabaja en un espacio geográfico limitado y seguro, dentro de un contexto tecnológico y hasta económico. Este tipo de modelo está representado por clases equipadas con dispositivos, donde los estudiantes, equipos y experiencia de aprendizaje están ubicados en un único espacio físico y no se permite la movilidad.

- Tipo 2. Dispositivos en clase, los estudiantes se mueven y comparten, pero la experiencia no es móvil. La agenda incluye el desarrollo de destrezas para el siglo XXI y la alfabetización digital. En cuanto a este tipo, se muestra una tendencia hacia la adopción de modelo BYOD y la integración de la tecnología.

- Tipo 3. Dispositivos, educandos y experiencias totalmente móviles. Se basa en la pedagogía y el desarrollo de destrezas para el siglo XXI y en la justicia social. En esta agenda se integra la alfabetización digital con otros aprendizajes, el proceso centrado en un estudiante activo, se potencia la colaboración, el aprendizaje es situado, integrado e inmersivo. Un ejemplo del tipo 3 es, según Pegrum (2014), la experiencia llevada a cabo en Singapur, con realidad aumentada, en donde los estudiantes siguen un recorrido específico en la ciudad $y$, a medida que se movilizan, el sistema hace preguntas, les pide respuestas, brinda y comparte información. Este es un caso de cómo el ambiente puede proveer información y conocimiento.

\section{- Producción de materiales para tecnologías móviles}

Cuando se realizan propuestas de uso de dispositivos móviles, en contextos educativos, se hace necesario planificar los recursos que estarán a disposición de los estudiantes, a través de estos medios. El estudio, realizado por Contreras, Herrera y Ramírez (2009), determinó que existen tres elementos instruccionales para la elaboración de materiales educativos móviles: los tecnológicos, los pedagógicos y los de diseño.

En cuanto a los elementos tecnológicos, estos están referidos a la interfaz de navegación intuitiva, la adaptabilidad y los protocolos. En este elemento deben ubicarse los aspectos implicados con posibilidades de acceso, la seguridad, así como la infraestructura y herramientas que dan soporte al proyecto. Una de las recomendaciones de los investigadores fue considerar las posibilidades de descargar los materiales, para no obligar al estudiante a la conectividad permanente para usarlos (Contreras, y otros, 2009).

Algunos de los aspectos específicos que deben considerarse, según Contreras y sus colegas, son:

- El tipo de aplicaciones: vídeos, audios, juegos, correo electrónico, navegador, reproductor, entre otros.

- La compatibilidad con los sistemas operativos.

- La red que transmita una señal y la convierta en datos o imágenes (topologías). 
- El tamaño de la pantalla, la adecuación de las imágenes a la medida del dispositivo.

- La seguridad informática.

- La accesibilidad web.

Por otro lado, en los elementos pedagógicos se hace necesario considerar: los objetivos educacionales definidos para el material a desarrollar, el fundamento o paradigma epistemológico en el cual se sustenta, el contexto de interacción, las actividades y la evaluación. Contreras y otros (2009), sugieren que los materiales tengan las características de objetos de aprendizaje: reutilizables e interoperables, de manera que puedan ser aprovechados en varios cursos y disciplinas.

Otro aspecto pedagógico a considerar, en la producción de materiales y las actividades asociadas a ellos, es el aprovechamiento del potencial de comunicación en red que tienen los dispositivos móviles. Las "Directrices de la Unesco para Políticas de Aprendizaje Móvil” (Unesco, 2013) indican que este aprendizaje puede ser útil para "acceder a recursos pedagógicos, conectarse con otras personas o crear contenidos, tanto fuera como dentro del aula" (p. 6), y abarca, también, los procesos administrativos eficaces y la mejora en la comunicación entre los actores educativos.

Akhshabi, Khalatbari y Akhshabi (2011) y la Unesco (2013) indican que las actividades instruccionales, llevadas a cabo a través de dispositivos móviles, deben buscar: acceder a materiales remotos; incrementar la cooperación entre los estudiantes; promover el aprendizaje en ambientes auténticos y reales fuera de las aulas; aprender de manera informal y a lo largo de la vida; incrementar el grado de alfabetización; hacer uso de las potencialidades del audiovisual para potenciar la comprensión; fortalecer la retención y comprensión de datos primordiales; mantener comunicación oportuna y efectiva con la clase o el grupo; propiciar una retroalimentación inmediata a los estudiantes; adjuntar fotografías o imágenes para proveer información más detallada sobre un hecho, proceso o fenómeno; dar prioridad al papel del estudiante en el proceso de aprendizaje: enviando mensajes, posteando imágenes, vídeos, compartiendo experiencias; y automatizar la distribución de documentos, y envío y evaluación de pruebas.

Finalmente, desde el punto de vista del diseño, es indispensable, en primera instancia, tomar en cuenta los criterios de un equipo interdisciplinario que lidere el proceso de planificación, organización, definición de la estructura y desarrollo; criterios en los cuales se deberán establecer aspectos en el formato de la publicación tanto de los audios como de los videos, sobre este punto, Contreras (2009) indica que se deben considerar: resolución de los vídeos, tipo de tomas de cámara, tipografía, el uso del color de acuerdo a los estándares para los diferentes medios, calidad del audio, la iconografía intuitiva, imágenes claras y acordes a la temática.

\section{- Experiencias en la implementación de aprendizaje móvil}

La implementación de procesos de enseñanza y aprendizaje, mediante el uso de tecnología móvil, ha sido adoptada de diferentes maneras alrededor del mundo. Esta sección expone algunas experiencias e investigaciones que se han generado y que es relevante revisar si se quieren gestionar proyectos de aprendizaje móvil.

Uno de los primeros proyectos de aprendizaje móvil en Latinoamérica, o que al menos tuvo mucho impacto, fue el One Laptop Per Child (OLPC), esta es una iniciativa que dio origen en el Massachusetts Institute of Technology Media Laboratory (MIT's Media Laboratory). Los principios que rigen el diseño de los dispositivos usados en el proyecto son: bajo costo, bajo consumo, poca energía y conectividad. El modelo inicial de One Laptop per Child (OLPC) fue la XO-1, conocida como la computadora verde, $y$ podía ser adquirida por cien dólares.

El primer país, en el mundo, que adoptó el proyecto OLPC, fue Uruguay. El gobierno uruguayo dotó a todas las escuelas primarias con una laptop o computadora portátil por niño. Le siguió Perú, que involucró un total de 8300 escuelas. Otros proyectos fueron desarrollados en Gaza, Afganistán, 
Haití, Etiopía y Mongolia (Usaid y mEducation Alliance, 2012).

Recientemente, OLPC desarrolló un nuevo dispositivo, la XO-3 Tablet. Al igual que su antecesor, la XO-1, esta es de plástico y su pantalla es semiflexible, lo cual garantiza alta durabilidad. Quizás, la mayor novedad de este modelo consistió en que podía ser cargada, no solo usando una manija, tal como se hacía con la XO-1, sino también usando un panel solar. Igualmente, su precio no superó los 100 dólares. Actualmente, se trabaja en el modelo XO-4 Touch.

En Tailandia, el modelo lanzado en el año 2012, y que tiene el apoyo del Ministerio de Educación, fue el de una tableta por niño (OTPC, One Tablet Per Child). Según especifica Pichaichannarong (2014), más de 2 millones de tabletas con contenidos fueron entregadas a los estudiantes de primer grado; los libros de texto se siguen usando junto con las tabletas, pero todas las escuelas del país están conectadas a Internet; la formación de docentes se realiza durante un año y más de 50 mil profesores la han recibido.

Según la Office of the Education Council, Ministry of Education, Thailand (2014), para el 2014, el sistema fue mejorado para cubrir, además, a los estudiantes de séptimo grado y en sus tabletas fueron instalados contenidos de matemática, ciencias, lenguaje tailandés, inglés y estudios sociales. Este mismo informe indica que, la National Statistical Office ha reportado que, para el 2013, el 73\% de la población contaba con un dispositivo móvil propio, que podía ser utilizado para el aprendizaje.

Otro esfuerzo realizado por la Unesco fue el proyecto Reading in the Mobile Era. El estudio fue realizado por West, Wood y Eskelinen (2014) y partió de la necesidad de alfabetizar a la población mundial, con el fin de lograr mejores resultados educativos. La ubicuidad y la creciente utilidad que se le asignado a la tecnología móvil, hacen proponer estrategias de uso para reducir la crisis mundial de analfabetismo. Este estudio se centra en países en desarrollo: Etiopía, Ghana, India, Kenia, Pakistán, Nigeria y Zimbabue.

En este estudio, se intentó entender mejor a las personas que hoy leen gracias al uso intensivo de dispositivos móviles como medios para acceder a la información y al conocimiento, con el fin de establecer modelos. Los resultados obtenidos muestran que las mujeres gastan más tiempo leyendo en dispositivos móviles que los hombres; la lectura móvil impacta positivamente a los niños, 1 de cada 3 lo usan para leer cuentos; la lectura móvil apela (y puede beneficiar) a los principiantes y semi-alfabetos, tanto adultos como adolescentes; las personas que leen en dispositivos móviles leen más, en términos totales, que en materiales impresos.

Se identificaron tres barreras; la primera es la falta de contexto pertinente, muchos lectores no quieren leer los clásicos o grandes obras en una pantalla. También la falta de conectividad, sobre todo en las zonas rurales y más remotas de los países en desarrollo. La tercera barrera es el costo, crucial aspecto para muchas poblaciones.

Las recomendaciones que brinda el equipo de investigadores son: enseñar a leer a las mujeres, a través de dispositivos móviles, se puede usar para eliminar el estigma de discriminación sobre ellas; crear contenidos más interesantes para hombres; crear portales de contenido adaptado para maestros; crear contenidos que interesen a nuevos alfabetos; incrementar la creación de aplicaciones de lectura; promover el compromiso de los gobiernos y los operadores móviles para apoyar los procesos de alfabetización y de lectura móvil; mejorar la infraestructura y que los gobiernos sean conscientes de la importancia para lograr mejores índices de alfabetización.

Por último, otro ejemplo a nivel latinoamericano fue Tabletas para Educar, en Colombia. En la conferencia dictada por Martha Castellanos (2014), se dieron a conocer algunos resultados encontrados en los grupos de estudiantes y maestros que están utilizando tabletas en los procesos educativos; a los que hace referencia son: la deserción y el ausentismo se han reducido, se ha aumentado el interés por las asignaturas y las practicas investigativas, y los maestros están enriqueciendo la práctica pedagógica.

Por otra parte, la Universidad de los Andes indica que, estos niños beneficiados en Colombia con este proyecto, tienen un $5 \%$ más de probabilidad de entrar a la educación superior, un 4,6 \% de lograr 
tener una labor remunerada, un $2 \%$ de aumento en los test de conocimiento y menos del $46 \%$ de posibilidades de deserción escolar (Castellanos, 2014).

\section{- Consideraciones políticas para el aprendizaje móvil}

Cuando se realizan proyectos que integran tecnologías en los contextos educativos, es necesario considerar la necesidad que se intenta satisfacer y el abordaje pedagógico que se realizará, de manera que no se limite a un enfoque tecnocentrista. Consideraciones relativas a las posibilidades de acceso, de equidad, pertinencia y uso responsable deben estar presentes en todo proyecto de este tipo. Por esta razón, organismos, como por ejemplo la Unesco, han realizado investigaciones que señalan los aspectos previos a considerar, por parte de los encargados o autoridades gubernamentales e institucionales. A continuación se especifican, en forma resumida, cada uno de ellos (Unesco, 2013).

Quizás, una de las primeras decisiones que deben tomarse, en una institución o en un país, es asegurar un marco de trabajo que permita implementar la innovación. Para esto es necesario crear políticas, si no las hay, o ampliar y actualizar las existentes, de manera que se asegure el uso máximo del potencial de los dispositivos móviles en educación. Estas medidas deben contemplar la asesoría en las inversiones y la propuesta pedagógica.

Asimismo, un proyecto debe asegurar que las personas encargadas de implementarlo tengan las competencias y los conocimientos necesarios para asegurar un nivel de calidad. Por ello, debe ser prioritario capacitar a los docentes, con el fin de que el potencial tecnológico sea aprovechado en la práctica educativa, impulsando la transformación y el mejoramiento de los procesos de enseñanza y de aprendizaje. Aunado a esto, deben abrirse las oportunidades para el aprendizaje en red o compartido, con el fin de que los docentes puedan divulgar sus experiencias.

Otro aspecto, necesario para la capacitación de los docentes, es considerar el contexto en el cual se va a desarrollar y su entorno inmediato, para esto, es menester que se usen tecnologías móviles. El punto central de esta política radica en que los procesos de formación continua lleguen al lugar y en el momento necesario. Si los docentes pueden capacitarse en su propio entorno, es más probable la puesta en práctica de los aprendizajes y su contextualización.

Por otra parte, los encargados de implementar el proyecto deben asegurar que los contenidos pedagógicos, creados o adaptados, puedan ser accedidos mediante dispositivos móviles, garantizando la pertinencia y la accesibilidad. Es importante señalar aquí que, el potencial multimedia que ofrecen los dispositivos móviles es una oportunidad para hacer llegar contenidos a los estudiantes y atender la diversidad. Aspectos que deben considerarse en esta política son: la apertura de licencias de los contenidos móviles, la creación de plataformas informáticas que faciliten la adaptación y creación de contenidos móviles, y la generación de normas que procuren la accesibilidad.

Todo lo mencionado anteriormente debe promover la igualdad de género, garantizando la satisfacción de las necesidades de aprendizaje. La política al respecto debe disminuir la brecha en la tenencia de dispositivos móviles entre hombres y mujeres, propiciando que ambos puedan desarrollar competencias en el uso de las tecnologías de información y comunicación (TIC) para aprender. Esto se logra si se amplía y mejora la conectividad en un marco de equidad, al mejorar la infraestructura tecnológica que hace posible el aprendizaje móvil. En la realización de esta política debe pensarse en sistemas de subsidio, tarifas diferenciadas, servicios comunitarios, entre otras estrategias para facilitar el acceso en escuelas, universidades, bibliotecas y en los hogares.

Siguiendo esta misma línea de equidad, se deben plantear estrategias que brinden acceso en condiciones de igualdad, aún a las personas que carecen de dispositivos móviles personales. Para lograr esto, se proponen tres alternativas: a) los gobiernos e instituciones proporcionan dispositivos a los estudiantes; b) los estudiantes aportan sus propios dispositivos, lo cual abre oportunidades de aprendizaje fuera del recinto institucional y c) la responsabilidad es compartida entre estudiantes, gobiernos e instituciones. 
De manera paralela a la implementación de tecnologías de las propuestas educativas, es urgente promover el uso responsable, adecuado y productivo de las tecnologías móviles; haciendo conciencia en los estudiantes de cuáles son los peligros a los que se exponen, dada la conectividad y comunicación que facilitan, y cuáles son las condiciones de seguridad para su operación. Esta acción también procurará establecer un equilibrio entre la interacción en línea y la vida real, y procurar usos saludables y productivos de la tecnología.

Sin duda alguna, los aspectos de gestión no deben omitirse al realizar proyectos de este tipo. Por ende, la tecnología móvil puede mejorar la gestión de la comunicación y la educación, simplificando tareas administrativas y proveyendo recursos para el aprendizaje.

Por último, para disminuir las barreras que impiden un uso generalizado del aprendizaje móvil, se debe aumentar la conciencia, mediante actividades de promoción, liderazgo y el diálogo. Para lograr esto, es necesario: compartir experiencias; compartir investigaciones de su aplicación en contextos educativos; alentar el diálogo entre los interesados; y tener una visión coherente sobre su inclusión y aporte, para el logro de los objetivos de aprendizaje.

\section{CONCLUSIONES}

Es innegable, como se ha expuesto, que la adopción de modelos pedagógicos, apoyados con tecnologías, implica retos y desafíos. En el caso de la UNED, los retos se desprenden de las realidades o condiciones que rodean al estudiante, el profesor y las tendencias mundiales. Estas pueden ser clasificadas en dos grandes categorías: aquellas que son propias de la adopción de tecnologías para responder a las exigencias de la sociedad, y aquellas que pueden garantizar una propuesta pedagógica pertinente y que derive en buenos resultados en el aprendizaje.

En cuanto a la adopción de la tecnología, es necesario tener en cuenta tres realidades: la tendencia a la adopción de dispositivos móviles en educación, la demanda actual de aplicaciones para dispositivos móviles y el aumento en la demanda de adquisición de tabletas, dadas sus capacidades lectoras.

En lo que concierne a la tendencia a la adopción de dispositivos móviles en educación, el Informe Horizon señala que las instituciones de educación superior deben adoptar, en menos de un año, el uso de aplicaciones para dispositivos móviles. Esto se fundamenta en que estos dispositivos han sido integrados a la vida diaria, por lo que los estudiantes de las universidades están expectantes acerca de la forma de utilizarlos para aprender (Johnson, y otros, 2012).

Referente a la segunda realidad, la demanda actual de aplicaciones para dispositivos móviles, el Informe de Distimo (Koekkoek, 2011) indica que el año 2011 fue "turbulento", dado el crecimiento que tuvo el desarrollo y disponibilidad de aplicaciones para móviles. En conjunto, todas las tiendas virtuales ofertaron más de un millón de aplicaciones. Este incremento se debe a que los dispositivos cuentan con características que permiten la captura, almacenamiento, procesamiento y transferencia de la información.

En cuanto al aumento en la demanda de adquisición de tabletas, el informe, de marzo del 2012 de Distimo (Spriensma, 2012) indicó que el primer iPad salió al mercado hace solo dos años y ya se habían vendido 50 millones. Pese a que posee un menor índice de venta, es evidente que muchos usuarios de tabletas las seleccionan por la capacidad lectora que poseen puesto que hay aplicaciones que permiten leer periódicos, revistas y libros electrónicos.

El panorama anterior obliga a la UNED, y a las instituciones educativas en general, a repensar los modelos pedagógicos y los contextos posibles de enseñanza y de aprendizaje, si se utilizan los dispositivos móviles.

Por otra parte, las realidades, que son inherentes a la propuesta pedagógica, señalan que los modelos de educación actuales presionan a las instituciones tradicionales a adoptar nuevos métodos de enseñanza, creativos y pertinentes, como la oferta de cursos en la Red, el uso de nuevas herramientas tecnológicas y la disposición de nuevos servicios a los estudiantes. De manera constante, emergen nuevos 
modelos educativos, apoyados en tecnologías que se posicionan a la vanguardia y como competencia de los modelos tradicionales; que, presionados por la situación económica, carecen de recursos humanos y financieros para generar cambios sustanciales de manera oportuna (Johnson y otros, 2012).

Aunado a lo anterior y debido al potencial tecnológico de los dispositivos móviles y a su uso en la vida cotidiana, se ha generado un aumento en las investigaciones acerca de cómo pueden coadyuvar estos en el currículo académico. Ya que las aplicaciones diseñadas para estos dispositivos han impactado en las disciplinas de la vida universitaria, a pesar de que no han sido creadas para este fin, están siendo adaptadas a las necesidades educativas (Johnson y otros, 2012).

Por último, como es posible verificar en las experiencias descritas con dispositivos móviles y en las propuestas de modelos que se han realizado para su implementación, hay desafíos que deben solventarse para poder implementar proyectos educativos apoyados en el uso de dispositivos móviles. Por ejemplo: la conectividad y la poca capacidad de almacenamiento, la producción de recursos educativos multiplataforma, el aprovechamiento del potencial de interacción y de movilidad de los dispositivos móviles, la creación de entornos de aprendizaje personalizados y colaborativos y la capacitación docente (Atchoarena , 2014; Marés, 2012; Nussbaum, 2014; Pegrum, 2013, 2014; Quimarou, 2014)

Se concluye entonces que la implementación de la innovación tecnológica tiene realidades a considerar y desafíos que deben ser atendidos para garantizar el éxito en los proyectos en ambientes de equidad, cobertura y excelencia académica. Por lo tanto, su implementación exige a la UNED un plan que cubra, no solo la adquisición de dispositivos, sino también la realización de recursos adaptables a ellos.

\section{REFERENCIAS}

Agüero, M. (2012). 44\% de ticos acceden a Internet desde el celular. Lunes 7 de mayo del 2012. Periódico La Nación, Sección El País.

Akhshabi, M., Khalatbari, J. y Akhshabi, M. (2011). An Experiment on Conducting Mobile Learning Activities on the Virtual University, Procedia - Social and Behavioral Sciences, Volume 28, 2011, Pages 384-389. Recuperado de: http://www.sciencedirect. com/science/article/pii/S1877042811025122

Atchoarena, D. (2014). Mobile for teachers development findings from UNESCO field projects in México, Nigeria, Pakistan and Senegal. Trabajo presentado en UNESCO Mobile Learning Week, Paris, France.

Burbules, N. (2010). Ubiquitous learning and the future of teaching.En memoria del XV Congreso de Tecnología y Educación a Distancia, Universidad Estatal a Distancia, San José, Costa Rica.

Castellanos,M. (2014). Tabletas para educar: Usos y experiencias innovadoras que contribuyen a fortalecer la calidad de la educación en Colombia. Trabajo presentado en UNESCO Mobile Learning Week, Paris, France.

Contreras, J. Herrera, J. y Ramírez, M. (2009). Elementos instruccionales para el diseño y la producción de materiales educativos móviles Revista Apertura - Vol. 1, núm. 1, octubre de 2009 http://www.udgvirtual.udg. mx/apertura/num11/REVISTA-ELECTRONICA/ Articulos\%20html/Articulo_7.html

Enríquez, L. y Chaos, L. (2006). Movilidad y educación: m-learning. Revista electrónica Entérate en línea: Internet, cómputo y telecomunicaciones. Año 5, Número 54. Universidad Nacional Autónoma de México. Recuperado el 07 de mayo de 2012 de: http://www.enterate.

unam.mx/Articulos/2006/noviembre/m-learning.htm

Galvan, J. (2006). Writing Literature Reviews. A guide for students of the social and behavioral sciences (3th. Ed.). Glendale, CA: Pyrczack Publishing. 
García, L., Ruiz, M. y Domínguez, D. (2007). De la educación a distancia a la educación virtual (1a. ed.). Barcelona, España: Editorial Ariel, S.A.

Jiménez, L; Cortés, C; Martin, L. y Lozano, C. (2007). Diseño e implementación de un prototipo funcional de M-Learning. Revista Proyectos de grado en Ingeniería en Sistemas: REDIS (103, ISSN: 0120-5919. En: http://www.acis.org.co/fileadmin/ Revista_103/13.pdf

Johnson, L., Adams, S., y Cummins, M. (2012). The NMC Horizon Report: 2012 Higher Education Edition. Austin, Texas: The New Media Consortium.

Jones, V. y Jo, J. (2004). Ubiquitous learning environment: An adaptive teaching system using ubiquitous technology. En: http://www.ascilite.org.au/conferences/ perth04/procs/jones.html

Koekkoek, H. (2011). Distimo Publication Full Year 2011. Distimo Publication. Recuperado el 21 de mayo de 2012 de: http://www.distimo.com/publications

Koekkoek, H. (febrero, 2012). The Amazon Appstore: Show Me the Money. Distimo Publication. Recuperado el 07 de mayo de 2012 de: http://www.distimo.com/ publications

Marés, L. (2012). Tablets en educación. Oportunidades $y$ desafíos en políticas uno a uno. Organización de Estados Iberoamericanos. Metas educativas 2021. Recuperado el 21 de mayo de http://redesoei.ning. com/video/tablets-en-educaci-n-oportunidades-ydesaf-os-en-pol-ticas-uno-1

Nussbaum, M. (2014). Learning from mistakes of the past and using technology to facilitate synergic scaffolding. Trabajo presentado en UNESCO Mobile Learning Week, Paris, France.

Office of the Education Council. Ministry of Education, Thailand (2014). Teacher Training and Support on Mobile Learning. How Thai Teachers Can Cope with the Unknown Terrain. Overview of Mobile Learning in Thailand.

Pegrum, M. (2013). Schools going mobile: A study of the adoption of mobile handheld technologies in Western Australian independent schools.
Australasian Journal of Educational Technology, 29(1), pp. 66-81

Pegrum, M. (2014). How can we balance affordability and affordance in the design of mobile pedagogy? Trabajo presentado en UNESCO Mobile Learning Week, Paris, France.

Pichaichannarong, S. (2014). Policy perspective: training and supporting teachers for mobile learning. Trabajo presentado en UNESCO Mobile Learning Week, Paris, France.

Quimarou, N. (2014). Teachers and mobile learning, voices from the ground. Trabajo presentado en UNESCO Mobile Learning Week, Paris, France.

Real Academia Española (2001). Diccionario de la Lengua Española. (22a.ed., Vol. 2). Colombia: ESPASA.

Roquet, G. (2008). Glosario de Educación a Distancia. Universidad Nacional Autónoma de México. Coordinación de Universidad Abierta y a Distancia, CUAED. Recuperado el 23 de abril del 2012 de: http://www.uned.es/catedraunesco-ead/varios/ Glosario.pdf

Smaldino, S., Lowther, D., Russell, J. (2007).Instructional Technology and Media for Learning (9 ${ }^{\text {th }}$. Ed.). UpperSaddleRiver, NJ: Prentice Hall.

Spriensma, G. (March, 2012). iPad - Two Years In Review. Distimo Publication. Recuperado de: http:// www.distimo.com/publications

UNESCO (2013). Directrices de la UNESCO para Políticas de Aprendizaje Móvil. Francia. Publicado por la Organización de las Naciones Unidas para la Educación, la Ciencia y la Cultura.

Universidad Estatal a Distancia (2004). Modelo Pedagógico. EUNED.

USAID and mEducation Alliance (February, 2012) 5 Minute Digest, a discussion with One Laptop per Child (OLPC) on the new XO 3 tablet and learning.

West, M., Wood, E., Eskelinen, S. (2014) Mobiles for reading findings from two soon to be published UNESCO reports. Trabajo presentado en UNESCO Mobile Learning Week, Paris, France. 
